\title{
Topological Derivative for Imaging of Thin Electromagnetic Inhomogeneity: Least Condition of Incident Directions
}

\author{
Won-Kwang Park (i) \\ Department of Information Security, Cryptology, and Mathematics, Kookmin University, Seoul, Republic of Korea \\ Correspondence should be addressed to Won-Kwang Park; parkwk@kookmin.ac.kr
}

Received 19 June 2017; Accepted 28 January 2018; Published 22 February 2018

Academic Editor: Antonio Scarfone

Copyright (C) 2018 Won-Kwang Park. This is an open access article distributed under the Creative Commons Attribution License, which permits unrestricted use, distribution, and reproduction in any medium, provided the original work is properly cited.

\begin{abstract}
It is well-known that using topological derivative is an effective noniterative technique for imaging of crack-like electromagnetic inhomogeneity with small thickness when small number of incident directions are applied. However, there is no theoretical investigation about the configuration of the range of incident directions. In this paper, we carefully explore the mathematical structure of topological derivative imaging functional by establishing a relationship with an infinite series of Bessel functions of integer order of the first kind. Based on this, we identify the condition of the range of incident directions and it is highly depending on the shape of unknown defect. Results of numerical simulations with noisy data support our identification.
\end{abstract}

\section{Introduction}

In this paper, we consider topological derivative [1] based imaging technique for thin, curve-like penetrable electromagnetic inhomogeneity with small thickness. Originally, this has been considered for shape optimization problems [2-7] and was then successfully combined with the level-set method (see [8-13]) for various inverse scattering problems. Surprisingly, throughout some researches [14, 15], it has been confirmed that topological derivative is also one of noniterative imaging techniques and very effective algorithm. However, as we can see [15-21], the most work has considered full-aperture problems.

Throughout results of numerical simulations, it has been confirmed that topological derivatives can be applied in limited-aperture problems, and an analysis in limitedaperture problems has been performed in [22]. In this interesting research, a relationship between topological derivative imaging function and an infinite series of Bessel functions of first kind has been established and, correspondingly, a sufficient condition of the range of incident directions for application has been identified theoretically. However, a least condition of application still remains unknown. Motivated by this fact, we identify a least condition of the range of incident directions for a successful application in limited-aperture inverse scattering problem and confirm this condition is highly depending on the unknown shape of thin inhomogeneity.

The remainder of paper is organized as follows. In Section 2, we survey two-dimensional direct scattering problem and topological derivative based imaging technique. In Section 3 , we investigate a least condition of the range of incident directions and discuss its properties. In Section 4, several results of numerical simulations with noisy data are presented in order to support our investigation. A brief conclusion is given in Section 5.

\section{Introduction to Direct Scattering Problem and Topological Derivative}

Let $\Omega \subset \mathbb{R}^{2}$ be a homogeneous domain with smooth boundary $\partial \Omega$ that contains a homogeneous thin inhomogeneity $\Gamma$ with a small thickness $2 h$. That is,

$$
\Gamma=\{\mathbf{x}+\eta \mathbf{n}(\mathbf{x}): \mathbf{x} \in \sigma,-h \leq \eta \leq h\},
$$

where $\mathbf{n}(\mathbf{x})$ is the unit normal to $\sigma$ at $x$ and $\sigma$ denotes the a simple, smooth curve in $\mathbb{R}^{2}$ which describes the supporting curve of $\Gamma$. In this contribution, we assume that the applied angular frequency is of the form $\omega=2 \pi f$. We assume that 
all materials are characterized by their dielectric permittivity and magnetic permeability at frequency of operation $\omega$; we define the piecewise constant permittivity $\varepsilon(\mathbf{x})$ and permeability $\mu(\mathbf{x})$ as

$$
\begin{aligned}
& \mathcal{\varepsilon}(\mathbf{x}):= \begin{cases}\varepsilon_{0} & \text { for } \mathbf{x} \in \Omega \backslash \bar{\Gamma} \\
\varepsilon_{\star} & \text { for } \mathbf{x} \in \Gamma,\end{cases} \\
& \mu(\mathbf{x}):= \begin{cases}\mu_{0} & \text { for } \mathbf{x} \in \Omega \backslash \bar{\Gamma} \\
\mu_{\star} & \text { for } \mathbf{x} \in \Gamma,\end{cases}
\end{aligned}
$$

respectively. For this sake, we set $\varepsilon_{0}=\mu_{0} \equiv 1$ and denote $k=\omega \sqrt{\varepsilon_{0} \mu_{0}}=2 \pi / \lambda$ as the wavenumber, where $\lambda$ is a given wavelength and satisfies $h \ll \lambda$.

Let $u^{(n)}(\mathbf{x} ; \omega)$ be the time-harmonic total field that satisfies Helmholtz equation

$$
\nabla \cdot\left(\frac{1}{\mu(\mathbf{x})} \nabla u^{(n)}(\mathbf{x} ; \omega)\right)+\omega^{2} \varepsilon(\mathbf{x}) u^{(n)}(\mathbf{x} ; \omega)=0
$$

$\mathbf{x} \in \Omega$

with boundary condition

$$
\frac{1}{\mu_{0}} \frac{\partial u^{(n)}(\mathbf{x} ; \omega)}{\partial \nu(\mathbf{x})}=\frac{1}{\mu_{0}} \frac{\partial e^{i \omega \boldsymbol{\theta}_{n} \cdot \mathbf{x}}}{\partial \nu(\mathbf{x})}, \quad \mathbf{x} \in \partial \Omega
$$

and with transmission conditions on the boundary of $\Gamma$. Here, $\boldsymbol{\theta}_{n}=\left[\cos \left(\theta_{n}\right), \sin \left(\theta_{n}\right)\right]$ denotes a two-dimensional vector on the connected, proper subset of unit circle $\mathbb{S}^{1}$ such that

$$
\theta_{n}=\theta_{1}+\frac{n-1}{N-1}\left(\theta_{N}-\theta_{1}\right), \quad 0<\theta_{N}-\theta_{1}<2 \pi
$$

Similarly, let $u_{\mathrm{B}}^{(n)}(\mathbf{x} ; \omega)=e^{i \omega \boldsymbol{\theta}_{n} \cdot \mathbf{x}}$ be the background solution of (3) with boundary condition (4).

Now, we introduce the basic concept of topological derivative operated at a fixed single frequency. The problem considered herein is the minimization of the tracking type functional depending on the solution $u^{(n)}(\mathbf{x} ; \omega)$ :

$$
\begin{aligned}
\mathbb{E}(\Omega ; \omega) & :=\frac{1}{2} \sum_{n=1}^{N}\left\|u^{(n)}(\mathbf{x} ; \omega)-u_{\mathrm{B}}^{(n)}(\mathbf{x} ; \omega)\right\|_{L^{2}(\partial \Omega)}^{2} \\
& =\frac{1}{2} \sum_{n=1}^{N} \int_{\partial \Omega}\left|u^{(n)}(\mathbf{x} ; \omega)-u_{\mathrm{B}}^{(n)}(\mathbf{x} ; \omega)\right|^{2} d S(\mathbf{x}) .
\end{aligned}
$$

Assume that an electromagnetic inclusion $\Sigma$ of small diameter $r$ is created at a certain position $\mathbf{z} \in \Omega \backslash \partial \Omega$, and let $\Omega \mid \Sigma$ denote this domain. Since the topology of the entire domain has changed, we can consider the corresponding topological derivative $d_{T} \mathbb{E}(\mathbf{z})$ based on $\mathbb{E}(\Omega)$ with respect to point $\mathbf{z}$ as

$$
d_{T} \mathbb{E}(\mathbf{z} ; \omega)=\lim _{r \rightarrow 0+} \frac{\mathbb{E}(\Omega \mid \Sigma ; \omega)-\mathbb{E}(\Omega ; \omega)}{\varphi(r ; \omega)},
$$

where $\varphi(r ; \omega) \rightarrow 0$ as $r \rightarrow 0+$. From (7), we can obtain an asymptotic expansion:

$$
\begin{aligned}
\mathbb{E}(\Omega \mid \Sigma ; \omega)= & \mathbb{E}(\Omega ; \omega)+\varphi(r ; \omega) d_{T} \mathbb{E}(\mathbf{z} ; \omega) \\
& +o(\varphi(r ; \omega)) .
\end{aligned}
$$

In [21], the following normalized topological derivative imaging function $\mathbb{E}_{\mathrm{TD}}(\mathbf{z} ; \omega)$ has been introduced:

$$
\begin{aligned}
& \mathbb{E}_{\mathrm{TD}}(\mathbf{z} ; \omega) \\
& \quad=\frac{1}{2}\left(\frac{d_{T} \mathbb{E}_{\varepsilon}(\mathbf{z} ; \omega)}{\max \left[d_{T} \mathbb{E}_{\varepsilon}(\mathbf{z} ; \omega)\right]}+\frac{d_{T} \mathbb{E}_{\mu}(\mathbf{z} ; \omega)}{\max \left[d_{T} \mathbb{E}_{\mu}(\mathbf{z} ; \omega)\right]}\right) .
\end{aligned}
$$

Here, $d_{T} \mathbb{E}_{\varepsilon}(\mathbf{z} ; \omega)$ and $d_{T} \mathbb{E}_{\mu}(\mathbf{z} ; \omega)$ satisfying (8) for purely dielectric permittivity contrast $\left(\varepsilon \neq \varepsilon_{0}\right.$ and $\left.\mu=\mu_{0}\right)$ and magnetic permeability contrast $\left(\varepsilon=\varepsilon_{0}\right.$ and $\left.\mu \neq \mu_{0}\right)$ cases, respectively, are explicitly expressed as (see [21])

$$
\begin{aligned}
& d_{T} \mathbb{E}_{\varepsilon}(\mathbf{z} ; \omega)=\operatorname{Re} \sum_{n=1}^{N}\left(u_{\mathrm{A}}^{(n)}(\mathbf{z} ; \omega) \overline{u_{\mathrm{B}}^{(n)}(\mathbf{z} ; \omega)}\right), \\
& d_{T} \mathbb{E}_{\mu}(\mathbf{z} ; \omega)=\operatorname{Re} \sum_{n=1}^{N}\left(\nabla u_{\mathrm{A}}^{(n)}(\mathbf{z} ; \omega) \cdot \overline{\nabla u_{\mathrm{B}}^{(n)}(\mathbf{z} ; \omega)}\right),
\end{aligned}
$$

where $u_{\mathrm{A}}^{(n)}(\mathbf{x} ; \omega)$ satisfies the adjoint problem

$$
\begin{aligned}
\Delta u_{\mathrm{A}}^{(n)}(\mathbf{x} ; \omega)+\omega^{2} u_{\mathrm{A}}^{(n)}(\mathbf{x} ; \omega) & =0 \text { in } \Omega \\
\frac{\partial u_{\mathrm{A}}^{(n)}(\mathbf{x} ; \omega)}{\partial \nu(\mathbf{x})} & =u^{(n)}(\mathbf{x} ; \omega)-u_{\mathrm{B}}^{(n)}(\mathbf{x} ; \omega)
\end{aligned}
$$

on $\partial \Omega$

\section{Least Condition of Incident Directions}

In this section, we identify the least condition of incident directions for applying topological derivative. For this, we introduce the structure of $d_{T} \mathbb{E}_{\varepsilon}(\mathbf{z} ; \omega)$ and $d_{T} \mathbb{E}_{\mu}(\mathbf{z} ; \omega)$ as follows.

Lemma 1 (see $[20,21])$. Suppose that $N$ and $\omega$ are sufficiently large; then

$$
\begin{aligned}
& d_{T} \mathbb{E}_{\varepsilon}(\mathbf{z} ; \omega) \approx \operatorname{Re} \sum_{n=1}^{N} \int_{\sigma}\left(\varepsilon_{\star}-\varepsilon_{0}\right) e^{i \omega \boldsymbol{\theta}_{n} \cdot(\mathbf{z}-\mathbf{x})} d \sigma(\mathbf{x}) \\
& d_{T} \mathbb{E}_{\mu}(\mathbf{z} ; \omega) \\
& \quad \approx \operatorname{Re} \sum_{n=1}^{N} \int_{\sigma} \Phi\left(\mathbf{t}(\mathbf{x}), \mathbf{n}(\mathbf{x}), \boldsymbol{\theta}_{n}\right) e^{i \omega \boldsymbol{\theta}_{n} \cdot(\mathbf{z}-\mathbf{x})} d \sigma(\mathbf{x}),
\end{aligned}
$$

where $\mathbf{t}(\mathbf{x})$ and $\mathbf{n}(\mathbf{x})$ are unit vectors that are, respectively, tangent and normal to the supporting curve $\sigma$ at $\mathbf{x}$, and

$$
\begin{aligned}
\Phi\left(\mathbf{t}(\mathbf{x}), \mathbf{n}(\mathbf{x}), \boldsymbol{\theta}_{n}\right)= & 2\left(\frac{1}{\mu_{\star}}-\frac{1}{\mu_{0}}\right) \boldsymbol{\theta}_{l} \cdot \mathbf{t}(\mathbf{x}) \\
& +2\left(\frac{1}{\mu_{0}}-\frac{\mu_{\star}}{\mu_{0}^{2}}\right) \boldsymbol{\theta}_{l} \cdot \mathbf{n}(\mathbf{x}) .
\end{aligned}
$$


Based on Lemma 1, the structure of (9) in limitedaperture problem can be represented as follows. This result plays an important role in identifying least condition of incident directions. For a detailed proof, we refer to [22].

Theorem 2. Let $\boldsymbol{\theta}=[\cos \theta, \sin \theta]^{T}$ and $\mathbf{x}-\mathbf{z}=$ $r[\cos \phi, \sin \phi]^{T}$. If $\omega$ and $N$ are sufficiently large, the structure of (9) becomes

$$
\begin{aligned}
& \mathbb{E}_{\mathrm{TD}}(\mathbf{z} ; \omega) \\
& \quad=\frac{1}{2}\left(\frac{\mathbb{W}_{1}(\mathbf{z}, \omega)}{\max \left[\mathbb{W}_{1}(\mathbf{z}, \omega)\right]}+\frac{\mathbb{W}_{2}(\mathbf{z}, \omega)}{\max \left[\mathbb{W}_{2}(\mathbf{z}, \omega)\right]}\right),
\end{aligned}
$$

where

$$
\begin{gathered}
\mathbb{W}_{1}(\mathbf{z}, \omega) \approx \int_{\sigma}\left(\varepsilon_{\star}-\varepsilon_{0}\right)\left(J_{0}(\omega|\mathbf{x}-\mathbf{z}|)\right. \\
\left.+\mathbb{D}\left(\omega|\mathbf{x}-\mathbf{z}|, \theta_{1}, \theta_{N}\right)\right) d \sigma(\mathbf{x})
\end{gathered}
$$

with

$$
\begin{aligned}
\mathbb{D} & \left(\omega|\mathbf{x}-\mathbf{z}|, \theta_{1}, \theta_{N}\right) \\
= & \frac{2}{\theta_{N}-\theta_{1}} \sum_{m=1}^{\infty} \frac{(-1)^{m}}{m} \sin \left\{m\left(\theta_{N}-\theta_{1}\right)\right\} \\
& \quad \times \cos \left\{m\left(\theta_{N}+\theta_{1}-2 \phi\right)\right\} J_{2 m}(\omega|\mathbf{x}-\mathbf{z}|)
\end{aligned}
$$

and the term $\mathbb{W}_{2}(\mathbf{z}, \omega)$ does not contribute to the imaging performance.

In recent work [20], it has been confirmed that the application of multifrequencies guarantees better a imaging performance than the application of a single frequency. Therefore, we consider the following normalized multifrequency topological derivative: for several frequencies $\left\{\omega_{f}\right.$ : $f=1,2, \ldots, F\}$, define

$$
\mathbb{E}(\mathbf{z} ; F):=\frac{1}{F} \sum_{f=1}^{F} \frac{d_{T} \mathbb{E}_{\mathrm{TD}}\left(\mathbf{z} ; \omega_{f}\right)}{\max \left[d_{T} \mathbb{E}_{\mathrm{TD}}\left(\mathbf{z} ; \omega_{f}\right)\right]} .
$$

Based on the structure of $\mathbb{E}_{\mathrm{TD}}(\mathbf{z} ; \omega)$ in Theorem 2, we can observe that the terms $J_{0}\left(\omega_{f}|\mathbf{x}-\mathbf{z}|\right)$ and $\mathbb{D}\left(\omega_{f} \mid \mathbf{x}-\right.$ $\left.\mathbf{z} \mid, \theta_{1}, \theta_{N}\right)$ contribute to and disturb the imaging performance, respectively, for $f=1,2, \ldots, F$. Hence, eliminating the term $\mathbb{D}\left(\omega_{f}|\mathbf{x}-\mathbf{z}|, \theta_{1}, \theta_{N}\right)$ will guarantee a good result. This means that the least condition comes from

$$
\begin{aligned}
& \sum_{m=1}^{\infty} \sin \left\{m\left(\theta_{N}-\theta_{1}\right)\right\} \\
& \quad \cdot \cos \left\{m\left(\theta_{N}+\theta_{1}-2 \phi\right)\right\} J_{2 m}\left(\omega_{f}|\mathbf{x}-\mathbf{z}|\right) \equiv 0
\end{aligned}
$$

for all $\mathbf{z} \in \Omega$ and $f=1,2, \ldots, F$. Note that since $J_{m}$ satisfies the asymptotic property

$$
J_{m}(x) \approx \sqrt{\frac{2}{x}} \cos \left(x-\frac{m \pi}{2}-\frac{\pi}{4}\right)
$$

for $x \gg\left|m^{2}-0.25\right|$, good results can appear in the map of $\mathbb{E}(\mathbf{z} ; F)$ when $\omega_{f} \approx+\infty$. Unfortunately, this is an ideal condition. Furthermore, since $\mathbf{z}$ is arbitrary, we cannot control the value of the term $J_{2 m}\left(\omega_{f}|\mathbf{x}-\mathbf{z}|\right)$. So, we must find a condition of $\theta_{1}$ and $\theta_{N}$ :

$$
\sin \left\{m\left(\theta_{N}-\theta_{1}\right)\right\} \cos \left\{m\left(\theta_{N}+\theta_{1}-2 \phi\right)\right\} \equiv 0
$$

for all $m \in \mathbb{N}$. A simple way is to select $\theta_{1}$ and $\theta_{N}$ such that

$$
\begin{array}{r}
\theta_{N}-\theta_{1}=\frac{\pi}{2}, \\
\theta_{N}+\theta_{1}-2 \phi=\frac{\pi}{2},
\end{array}
$$

that is, for any $\alpha \in \mathbb{R}$, a selection $\theta_{1}=\phi+\alpha$ and $\theta_{N}=\phi-\alpha+$ $(\pi / 2)$ will guarantee good results via topological derivative. Based on this, we can obtain the following theoretical result of the range of incident directions, which has been examined heuristically.

Theorem 3 (least condition of range). The least range of incident directions for successful application of topological derivative is highly depending on the shape of unknown thin inhomogeneity and the range of directions must be wider than $\pi / 2$.

\section{Simulations Results and Discussions}

In this section, some results of numerical simulations are exhibited to support identified condition mentioned in previous section. The homogeneous domain $\Omega$ is chosen as a unit circle centered at the origin, and two supporting curves $\sigma_{j}$ of the $\Gamma_{j}$ are selected as

$$
\begin{aligned}
& \sigma_{1}=\left\{[s, 0.2]^{T}:-0.5 \leq s \leq 0.5\right\} \\
& \sigma_{2}=\left\{\left[s+0.2, s^{3}+s^{2}-0.6\right]^{T}:-0.5 \leq s \leq 0.5\right\} .
\end{aligned}
$$

The thickness $h$ of all $\Gamma_{j}$ is set to 0.02 , and parameters $\varepsilon_{0}$, $\mu_{0}$ are chosen as 1 . Let $\varepsilon_{j}$ and $\mu_{j}$ for $j=1,2,3$ denote the permittivity and permeability of $\Gamma_{j}$, respectively. The applied frequency is selected as $\omega_{f}=2 \pi / \lambda_{f}, f=1,2, \ldots, F(=10)$ with $\lambda_{1}=0.7, \lambda_{10}=0.4$, and $N=16$ different incident directions chosen. In order to show the robustness, a white Gaussian noise with $20 \mathrm{~dB}$ signal-to-noise ratio (SNR) was added to the unperturbed boundary data.

First, let us consider the imaging of straight line shaped thin inhomogeneity $\Gamma_{1}$. In this case, since $\phi \equiv 0$, when $\mathbf{x}, \mathbf{z} \in$ $\Gamma_{1}, \theta_{1}=\alpha$ and $\theta_{N}=\alpha+(\pi / 2)$ will be a good choice for any $\alpha$. Corresponding results for $\alpha=0, \alpha=\pi / 6, \alpha=\pi / 4$, and $\alpha=3 \pi / 4$ are exhibited in Figure 1. Based on these results, we can observe that, for any value of $\alpha$, the shape of $\Gamma_{1}$ was retrieved satisfactorily via $\mathbb{E}(\mathbf{z} ; F)$ with the $\pi / 2$ range of incident directions. However, although one can recognize the existence of inhomogeneity, the shape of $\Gamma_{1}$ cannot be reconstructed satisfactorily when $\alpha=3 \pi / 4$. 


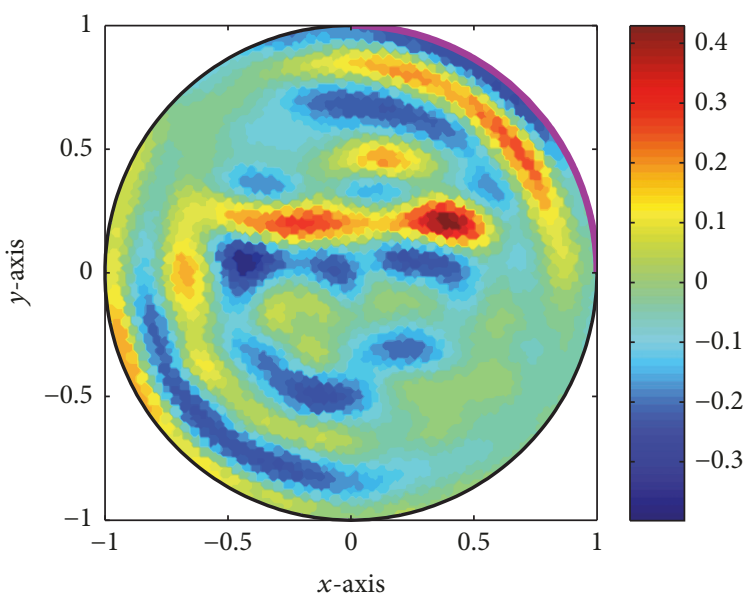

(a) $\theta_{1}=0$ and $\theta_{2}=\pi / 2$

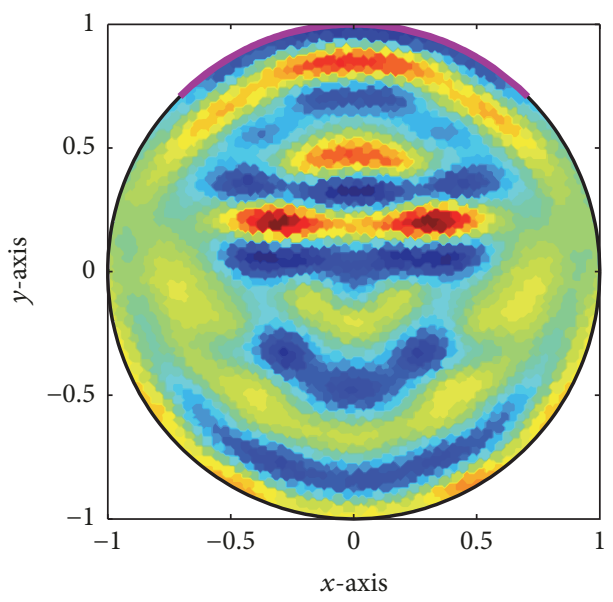

(c) $\theta_{1}=\pi / 4$ and $\theta_{2}=3 \pi / 4$

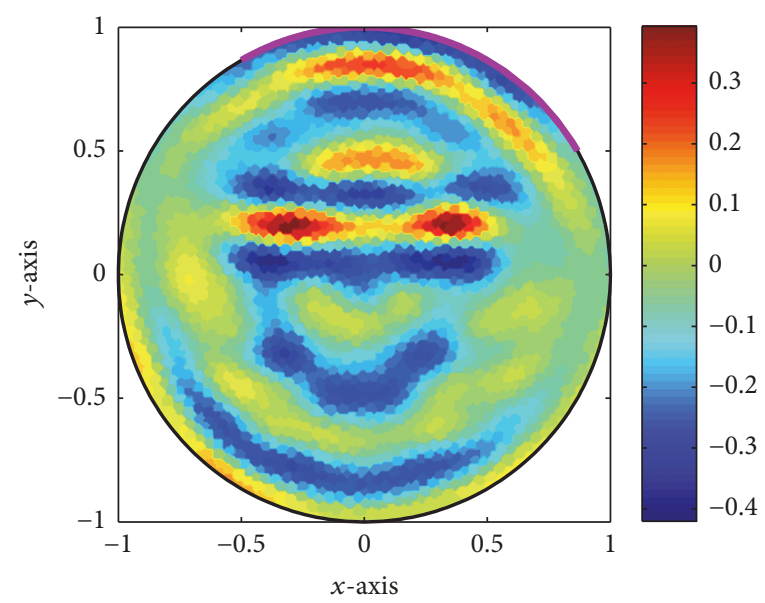

(b) $\theta_{1}=\pi / 6$ and $\theta_{2}=2 \pi / 3$

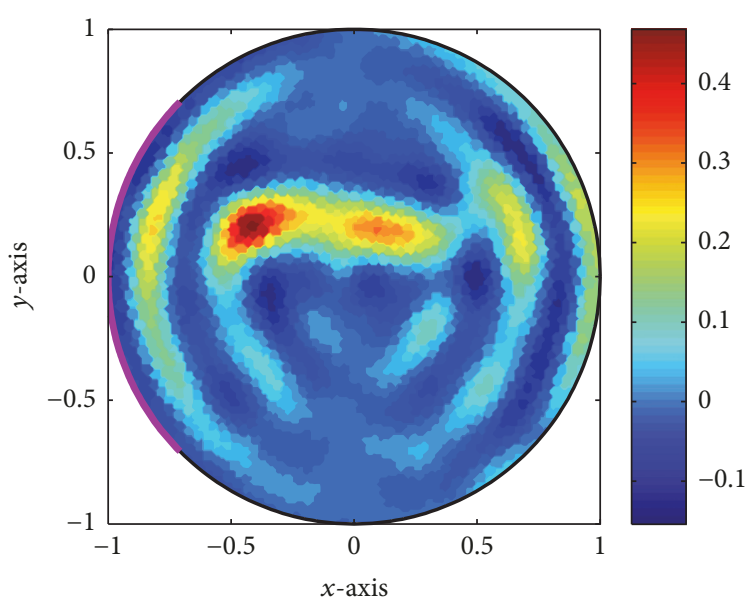

(d) $\theta_{1}=\pi / 2$ and $\theta_{2}=\pi$

Figure 1: Maps of $\mathbb{E}(\mathbf{z} ; F)$ for $\Gamma_{1}$. Violet-colored solid line describes the range of incident directions.

Based on the results in Figure 1, we can conclude that one can identify the shape of existence of $\Gamma_{1}$ for any choice of $\alpha$. But if the values of $\theta_{1}$ and $\theta_{N}$ are satisfying

$$
\begin{array}{r}
\theta_{1}=\xi_{\mathbf{t}}-\frac{\pi}{4}, \\
\theta_{N}=\xi_{\mathbf{t}}+\frac{\pi}{4},
\end{array}
$$

$$
\text { where } \mathbf{t}(\mathbf{x})=\left[\cos \left(\xi_{\mathbf{t}}\right), \sin \left(\xi_{\mathbf{t}}\right)\right]^{T},
$$

the result via the map of $\mathbb{E}(\mathbf{z} ; F)$ is very poor. This is the worst choice of the selection. In contrast, if the values of $\theta_{1}$ and $\theta_{N}$ are satisfying

$$
\begin{aligned}
\theta_{1} & =\xi_{\mathbf{n}}-\frac{\pi}{4}, \\
\theta_{N} & =\alpha+\frac{\pi}{4},
\end{aligned}
$$

an acceptable result should be obtained via the map of $\mathbb{E}(\mathbf{z} ; F)$; refer to Figure 2. With this observation, we can conclude that if $\theta_{1}$ and $\theta_{N}$ are satisfying

$$
\begin{aligned}
0 & \leq \theta_{1} \leq \xi_{\mathbf{n}}-\frac{\pi}{4}, \\
\xi_{\mathbf{n}}+\frac{\pi}{4} & \leq \theta_{N} \leq 2 \pi,
\end{aligned}
$$

a good result of $\Gamma_{1}$ can be obtained; refer to Figure 3 .

Now, let us consider the influence of range of incident directions when the shape of thin inhomogeneity is no more straight line. For this purpose, we choose thin inclusion $\Gamma_{2}$ and compare maps of $\mathbb{E}(\mathbf{z} ; F)$ for various range of incident directions. Figure 4 shows maps of $\mathbb{E}(\mathbf{z} ; F)$ for

$$
\begin{aligned}
\theta_{1} & =\frac{\pi}{4}-\alpha, \\
\theta_{N} & =\frac{\pi}{4}+\alpha,
\end{aligned}
$$

where $\alpha=\pi / 12, \pi / 6, \pi / 4$, and $\pi / 2$. Based on these result, we can observe that if the range of directions is narrow, we 


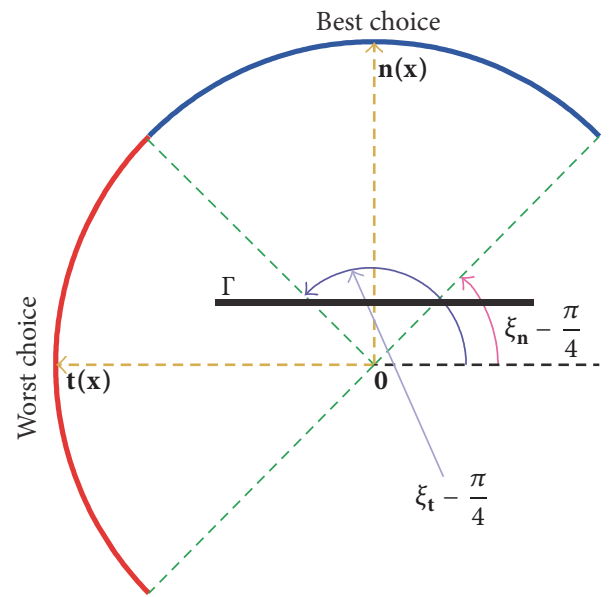

FIGURE 2: Description of best (blue-colored range) and worst (red-colored range) choice of the range of incident directions.

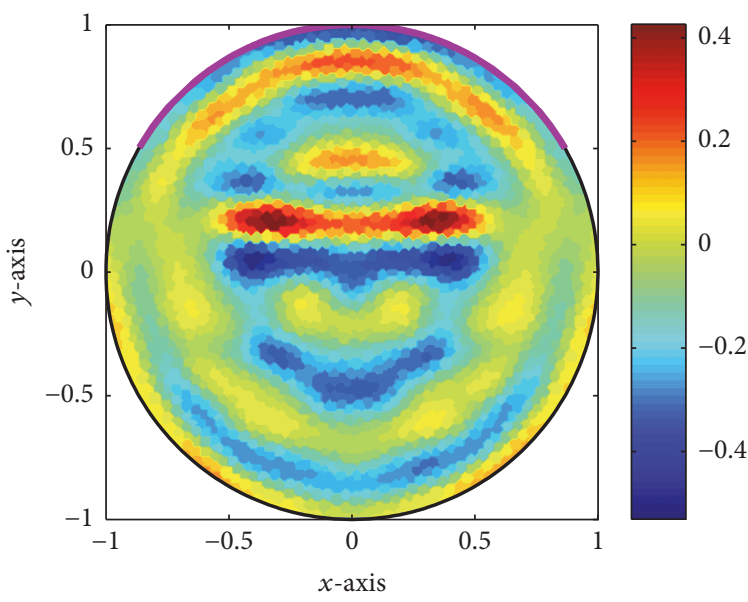

(a) $\theta_{1}=\pi / 6$ and $\theta_{2}=5 \pi / 6$

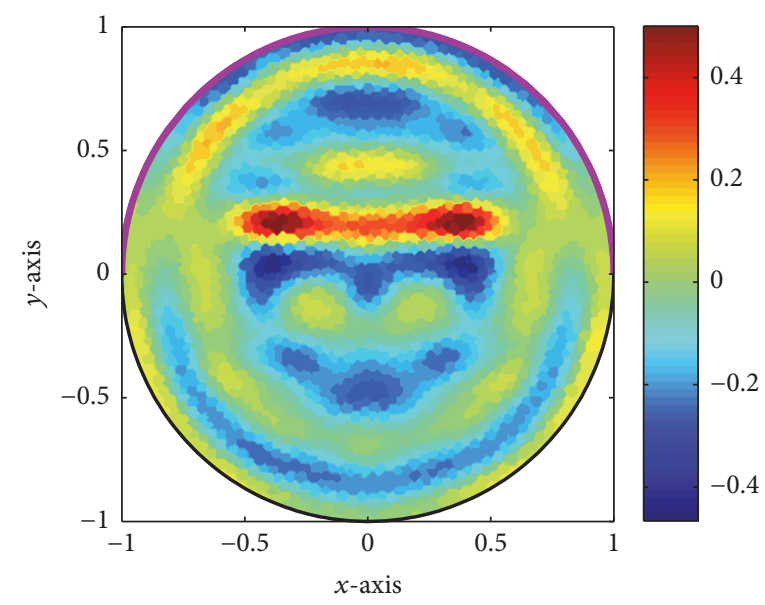

(b) $\theta_{1}=0$ and $\theta_{2}=\pi$

FIGURE 3: Same as Figure 1 except the range of the incident directions.

cannot recognize the existence of $\Gamma_{2}$; refer to Figures 4(a) and $4(\mathrm{~b})$. Note that when the range of directions satisfies the sufficient condition in [22], the shape of $\Gamma_{2}$ can be identified; refer to Figure 4(d). However, if one selects the optimal range, adopted for imaging of $\Gamma_{1}$, the result is still poor (see Figure 4(c)).

In order to find the least condition, let us reconsider the imaging of $\Gamma_{1}$. In this case, the selection of (24) was a good choice. Following this observation, one of the possible choices of $\alpha$ is that, for $\mathbf{n}(\mathbf{x})=\left[\cos \left(\xi_{\mathbf{x}}\right), \sin \left(\xi_{\mathbf{x}}\right)\right]^{T}, \mathbf{x} \in \sigma_{2}$, select

$$
\begin{aligned}
\theta_{1} & =\min \left\{\xi_{\mathbf{x}}: \mathbf{x} \in \sigma_{2}\right\}-\frac{\pi}{4}, \\
\theta_{N} & =\max \left\{\xi_{\mathbf{x}}: \mathbf{x} \in \sigma_{2}\right\}+\frac{\pi}{4} .
\end{aligned}
$$

Then, it is expected that identified shape of inhomogeneity will be close to the shape of $\Gamma_{2}$. Notice that, throughout the numerical computation,

$$
\begin{aligned}
\theta_{N}-\theta_{1} & =\max \left\{\xi_{\mathbf{x}}: \mathbf{x} \in \sigma_{2}\right\}-\min \left\{\xi_{\mathbf{x}}: \mathbf{x} \in \sigma_{2}\right\}+\frac{\pi}{2} \\
& <\pi
\end{aligned}
$$

Thus, selection of (27) is the least condition of the range of incident directions. Figure 6 exhibits maps of $\mathbb{E}(\mathbf{z} ; F)$ for $\theta_{1}$ and $\theta_{N}$ in (27) and for $\theta_{1}=0$ and $\theta_{N}=2 \pi$ (see Figure 5 for instance). Since these conditions satisfy least condition, the shape of $\Gamma_{2}$ seems retrieved well, and this result supports Theorem 3.

For the final example, let us consider the imaging of two, nonoverlapped thin inhomogeneities $\Gamma_{2}$ and $\Gamma_{3}$, where the supporting curve $\sigma_{3}$ of $\Gamma_{3}$ is

$$
\sigma_{3}=\left\{\left[s-0.2,-0.5 s^{2}+0.5\right]^{T}:-0.5 \leq s \leq 0.5\right\}
$$

and $\varepsilon_{2}=\varepsilon_{3}=\mu_{2}=\mu_{3}=5$. Based on the results in Figure 7, we can conclude that it is hard to recognize the shape of $\Gamma_{2} \cup \Gamma_{3}$ when the range $\theta_{N}-\theta_{1}<\pi$, but we can identify when 


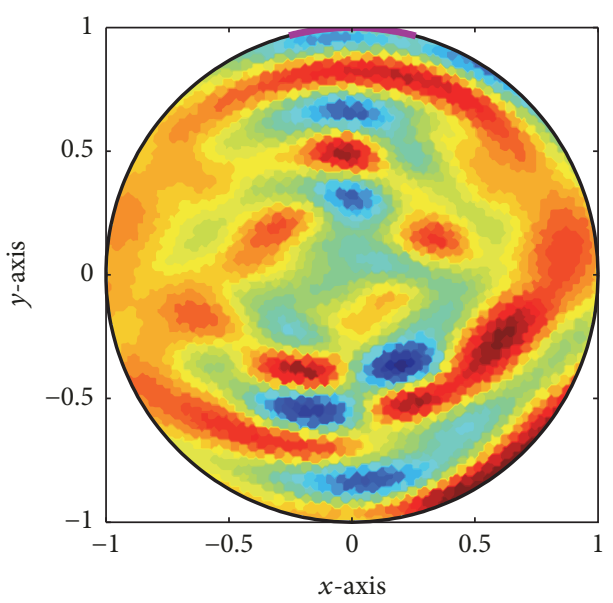

(a) $\theta_{1}=5 \pi / 12$ and $\theta_{2}=7 \pi / 12$

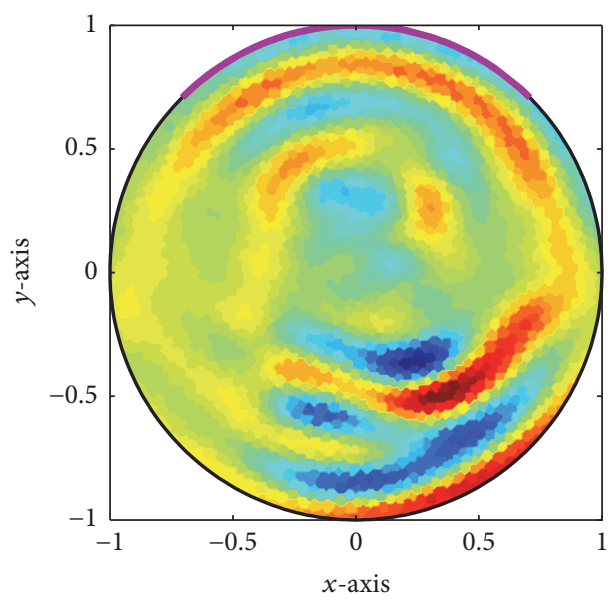

(c) $\theta_{1}=\pi / 4$ and $\theta_{2}=3 \pi / 4$
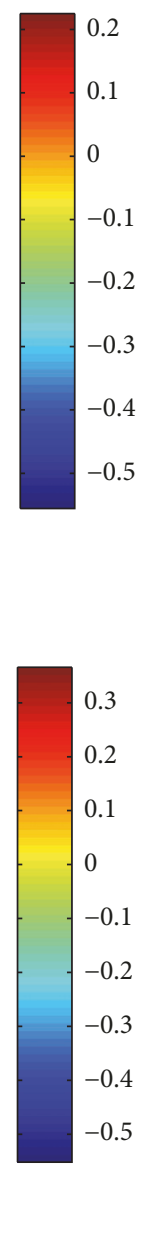

Figure 4: Maps of $\mathbb{E}(\mathbf{z} ; F)$ for $\Gamma_{1}$. Violet-colored solid line describes the range of incident directions.

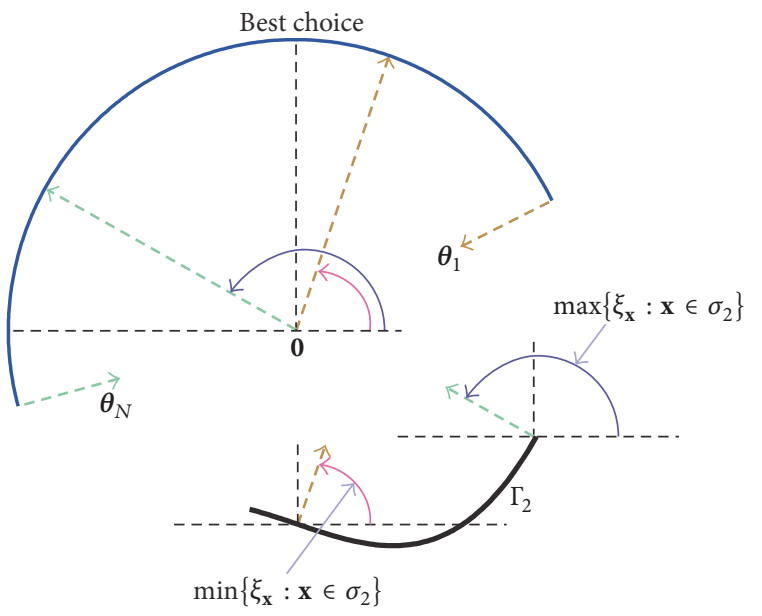

Figure 5: Description of best choice of the range of incident directions.

$\theta_{N}-\theta_{1} \approx \pi$. With this, we end up this section with the following remark.

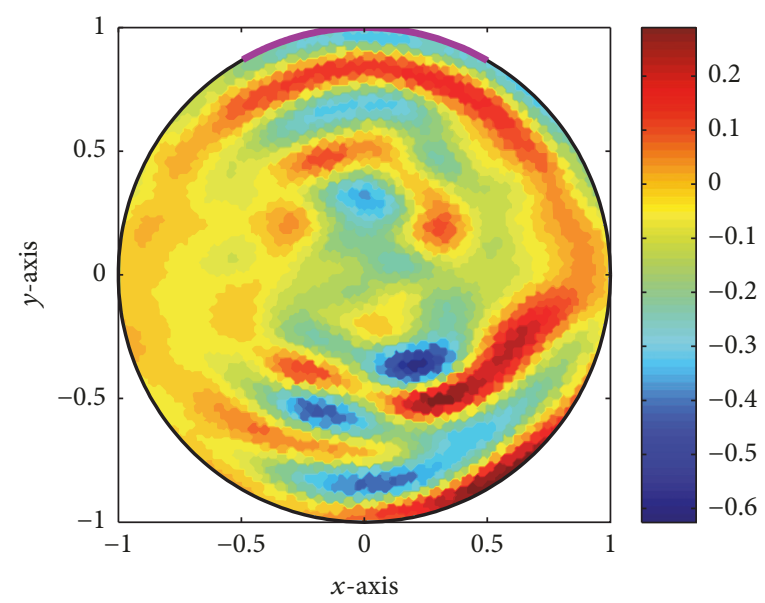

(b) $\theta_{1}=\pi / 3$ and $\theta_{2}=2 \pi / 3$

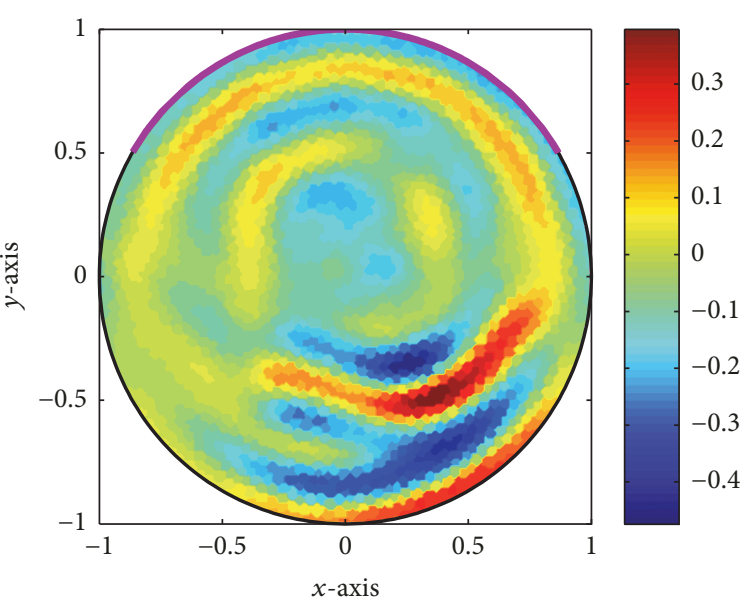

(d) $\theta_{1}=\pi / 3$ and $\theta_{2}=5 \pi / 3$

\section{Conclusion}

Remark 4 (condition for imaging of multiple inhomogeneities). Due to the shape dependency of the range of directions, when the shapes of thin inhomogeneities are not straight line, the range of directions must be close to $\pi$, which is the sufficient condition of range of application. Related results of numerical simulations can be found in [22] also.

In this paper, we have considered the topological derivative in a limited-aperture inverse scattering problem for a noniterative imaging of thin inhomogeneity. Based on the relationship between topological derivative imaging function and infinite series of Bessel functions of integer order of the first kind, we discovered a least condition of the range of incident directions for successful application. We presented the results of some numerical simulations, which show that the discovered condition is valid for the imaging of a thin inclusion. Here, we have considered an imaging of thin penetrable inhomogeneity but the analysis could be carried out for a perfectly conducting crack. Furthermore, the extension to inverse elasticity problems will be an interesting 


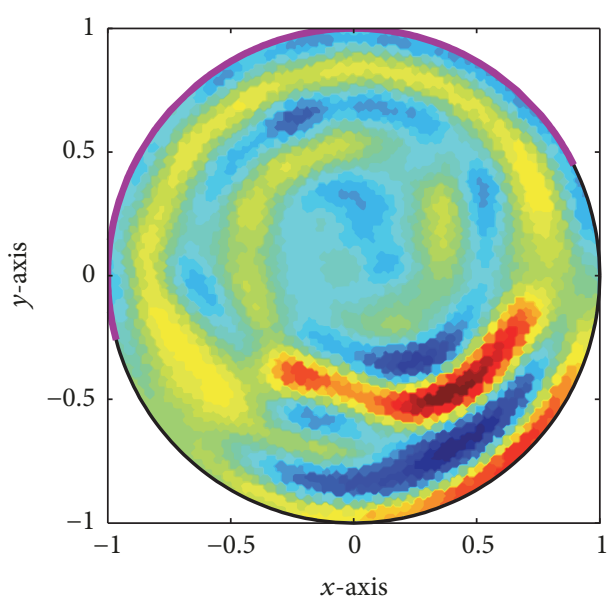

(a) $\theta_{1}$ and $\theta_{N}$ satisfy (27)

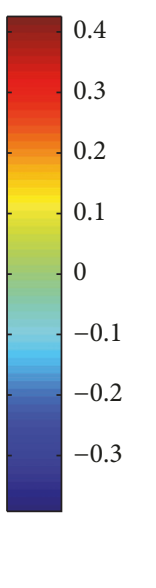

(

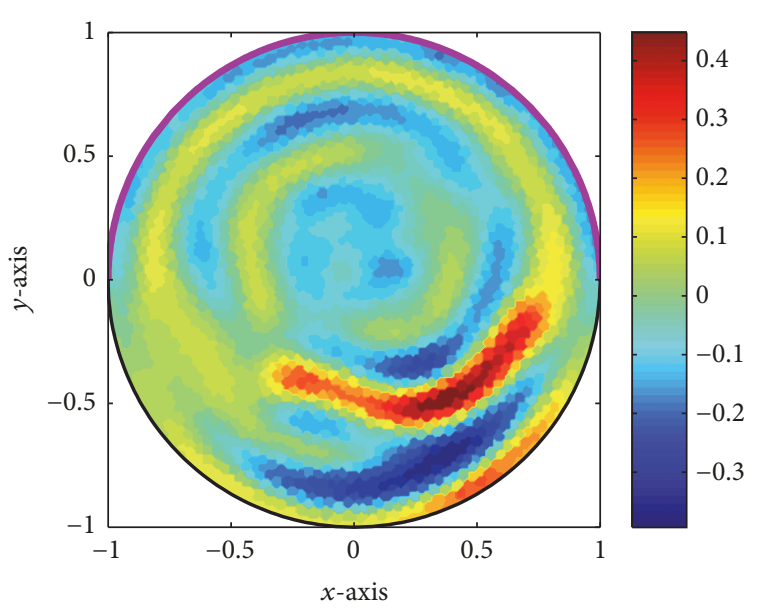

(b) $\theta_{1}=0$ and $\theta_{N}=\pi$

Figure 6: Map of $\mathbb{E}(\mathbf{z} ; F)$ for when inhomogeneity is $\Gamma_{2}$.

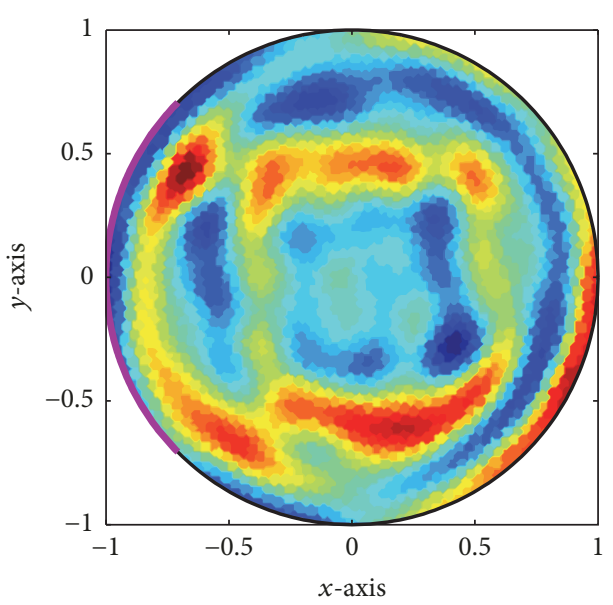

(a) $\theta_{1}=\pi / 2$ and $\theta_{2}=\pi$

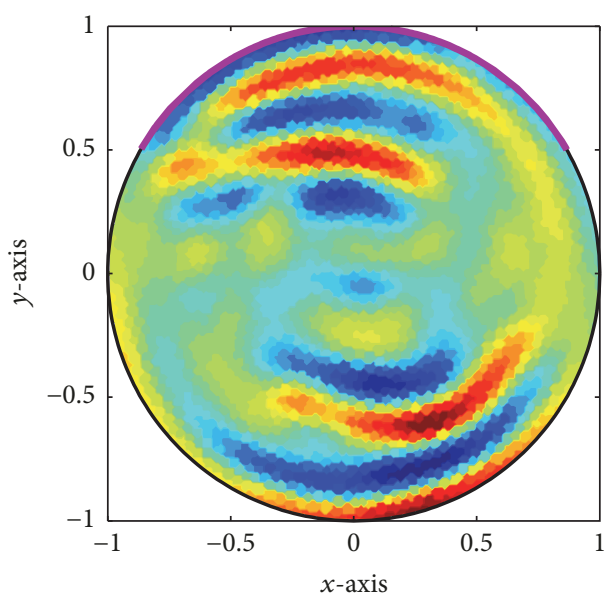

(c) $\theta_{1}=\pi / 6$ and $\theta_{2}=5 \pi / 6$
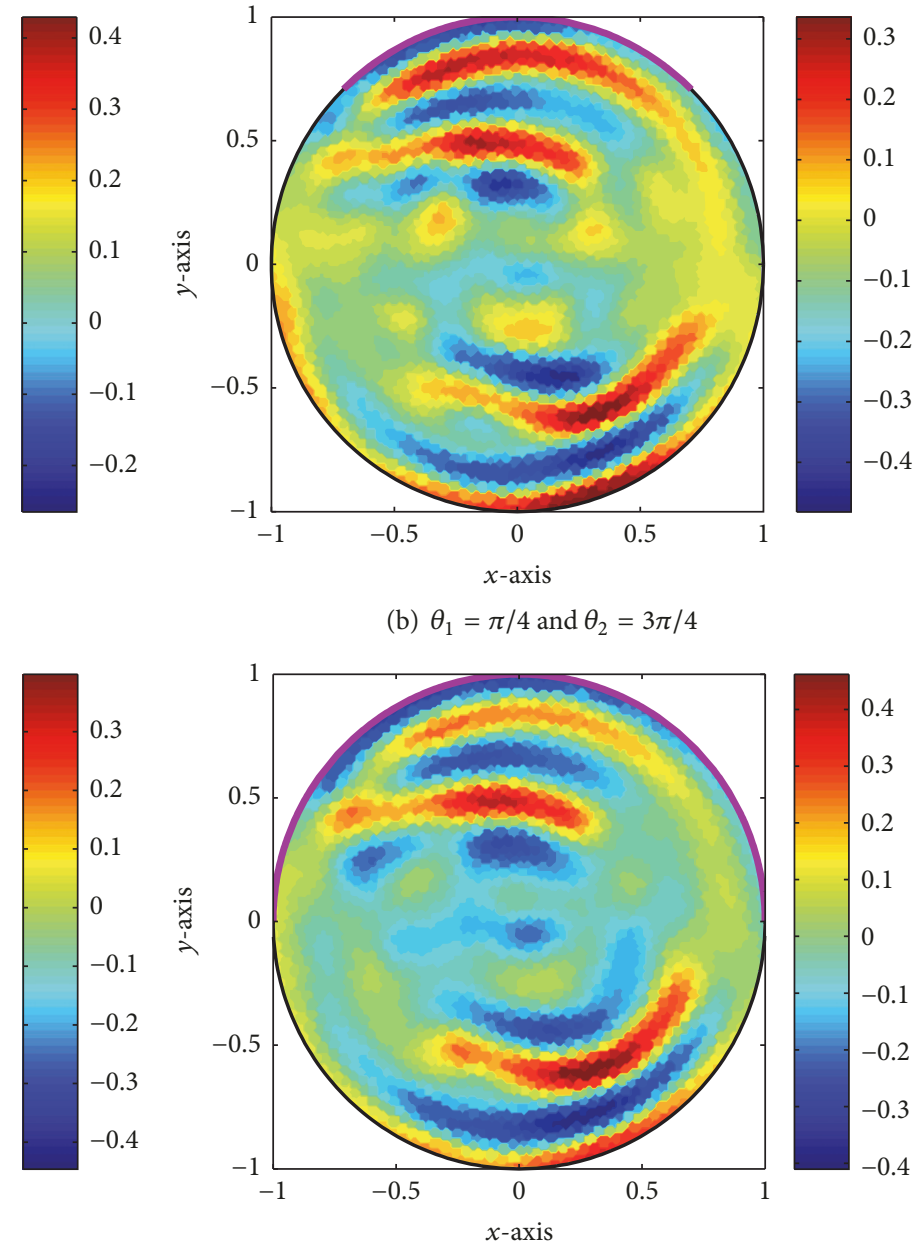

(d) $\theta_{1}=0$ and $\theta_{2}=\pi$

FIgURE 7: Maps of $\mathbb{E}(\mathbf{z} ; F)$ for $\Gamma_{2} \cup \Gamma_{3}$. Violet-colored solid line describes the range of incident directions. 
subject. Finally, extension to the three-dimensional $[23,24]$ and real-world problem $[25,26]$ will be a remarkable research topic.

\section{Conflicts of Interest}

The author declares that there are no conflicts of interest regarding the publication of this paper.

\section{Acknowledgments}

This research was supported by the Basic Science Research Program through the National Research Foundation of Korea (NRF) funded by the Ministry of Education (no. NRF2017R1D1A1A09000547) and the research program of Kookmin University in Korea.

\section{References}

[1] A. A. Novotny and J. Sokolowski, Topological derivatives in shape optimization, Interaction of Mechanics and Mathematics, Springer, Heidelberg, Berlin, 2013.

[2] A. Carpio and M.-L. Rapún, "Solving inhomogeneous inverse problems by topological derivative methods," Inverse Problems, vol. 24, no. 4, Article ID 045014, 2008.

[3] J. Céa, S. Garreau, P. Guillaume, and M. Masmoudi, “The shape and topological optimizations connection," Computer Methods Applied Mechanics and Engineering, vol. 188, no. 4, pp. 713-726, 2000.

[4] H. A. Eschenauer, V. V. Kobelev, and A. Schumacher, "Bubble method for topology and shape optimization of structures," Journal of Structural Optimization, vol. 8, no. 1, pp. 42-51, 1994.

[5] G. R. Feijoo, "A new method in inverse scattering based on the topological derivative," Inverse Problems, vol. 20, no. 6, pp. 1819$1840,2004$.

[6] J. Sokołowski and A. Zochowski, "On the topological derivative in shape optimization," SIAM Journal on Control and Optimization, vol. 37, no. 4, pp. 1251-1272, 1999.

[7] H. Yuan, G. Bracq, and Q. Lin, "Inverse acoustic scattering by solid obstacles: topological sensitivity and its preliminary application," Inverse Problems in Science and Engineering, vol. 24, no. 1, pp. 92-126, 2016.

[8] D. Álvarez, O. Dorn, N. Irishina, and M. Moscoso, "Crack reconstruction using a level-set strategy," Journal of Computational Physics, vol. 228, no. 16, pp. 5710-5721, 2009.

[9] M. Burger, B. Hackl, and W. Ring, "Incorporating topological derivatives into level set methods," Journal of Computational Physics, vol. 194, no. 1, pp. 344-362, 2004.

[10] O. Dorn and D. Lesselier, "Level set methods for inverse scattering," Inverse Problems, vol. 22, no. 4, article no. R01, pp. R67-R131, 2006.

[11] L. He, C.-Y. Kao, and S. Osher, "Incorporating topological derivatives into shape derivatives based level set methods," Journal of Computational Physics, vol. 225, no. 1, pp. 891-909, 2007.

[12] W.-K. Park, "Shape reconstruction of thin electromagnetic inclusions via boundary measurements: level-set method combined with the topological derivative," Mathematical Problems in Engineering, vol. 2013, Article ID 125909, 2013.
[13] W.-K. Park and D. Lesselier, "Reconstruction of thin electromagnetic inclusions by a level-set method," Inverse Problems, vol. 25, no. 8, Article ID 085010, 2009.

[14] H. Ammari, H. Kang, H. Lee, and W.-K. Park, "Asymptotic imaging of perfectly conducting cracks," SIAM Journal on Scientific Computing, vol. 32, no. 2, pp. 894-922, 2010.

[15] M. Bonnet, B. Guzina, and N. Nemitz, "Fast non-iterative methods for defect identification," Revue européenne de mécanique numérique, vol. 17, no. 5-6-7, pp. 571-582, 2008.

[16] H. Ammari, J. Garnier, V. Jugnon, and H. Kang, "Stability and resolution analysis for a topological derivative based imaging functional," SIAM Journal on Control and Optimization, vol. 50, no. 1, pp. 48-76, 2012.

[17] M. Bonnet, "Fast identification of cracks using higher-order topological sensitivity for 2-D potential problems," Engineering Analysis with Boundary Elements, vol. 35, no. 2, pp. 223-235, 2011.

[18] M. Jleli, B. Samet, and G. Vial, “Topological sensitivity analysis for the modified Helmholtz equation under an impedance condition on the boundary of a hole," Journal de Mathématiques Pures et Appliquées, vol. 103, no. 2, pp. 557-574, 2015.

[19] A. Malcolm and B. Guzina, "On the topological sensitivity of transient acoustic fields," Wave Motion, vol. 45, no. 6, pp. 821834, 2008.

[20] W.-K. Park, "Multi-frequency topological derivative for approximate shape acquisition of curve-like thin electromagnetic inhomogeneities," Journal of Mathematical Analysis and Applications, vol. 404, no. 2, pp. 501-518, 2013.

[21] W.-K. Park, “Topological derivative strategy for one-step iteration imaging of arbitrary shaped thin, curve-like electromagnetic inclusions," Journal of Computational Physics, vol. 231, no. 4, pp. 1426-1439, 2012.

[22] C. Y. Ahn, K. Jeon, Y.-K. Ma, and W.-K. Park, "A study on the topological derivative-based imaging of thin electromagnetic inhomogeneities in limited-aperture problems," Inverse Problems, vol. 30, no. 10, Article ID 105004, 2014.

[23] S. Amstutz and N. Dominguez, "Topological sensitivity analysis in the context of ultrasonic non-destructive testing," Engineering Analysis with Boundary Elements, vol. 32, no. 11, pp. 936-947, 2008.

[24] F. Le Louër and M. a. Rapún, “Topological sensitivity for solving inverse multiple scattering problems in three-dimensional electromagnetism. Part I: one step method," SIAM Journal on Imaging Sciences, vol. 10, no. 3, pp. 1291-1321, 2017.

[25] W.-K. Park, H. P. Kim, K.-J. Lee, and S.-H. Son, "MUSIC algorithm for location searching of dielectric anomalies from S-parameters using microwave imaging," Journal of Computational Physics, vol. 348, pp. 259-270, 2017.

[26] S.-T. Yang and H. Ling, "Application of compressive sensing to two-dimensional radar imaging using a frequency-scanned microstrip leaky wave antenna," Journal of Electromagnetic Engineering and Science, vol. 17, no. 3, pp. 113-119, 2017. 


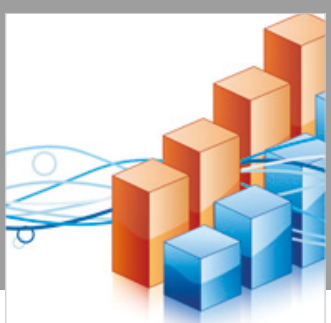

Advances in

Operations Research

\section{-n-m}
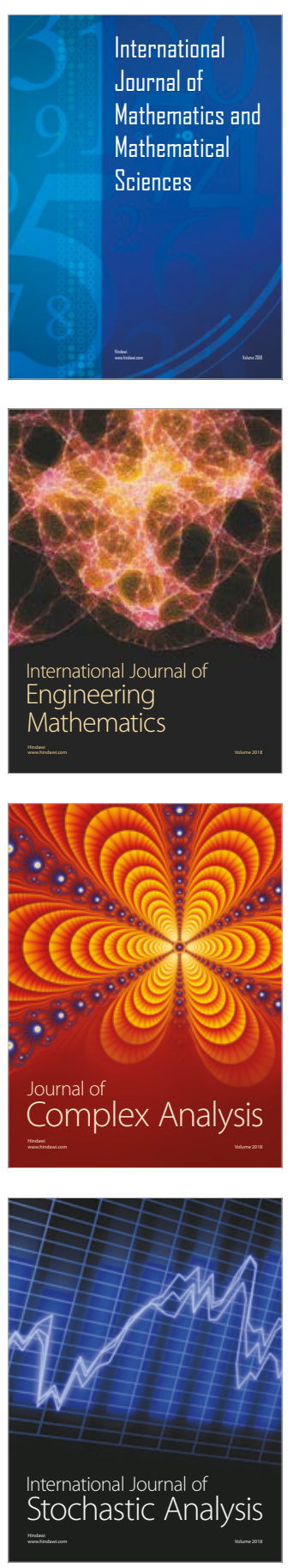
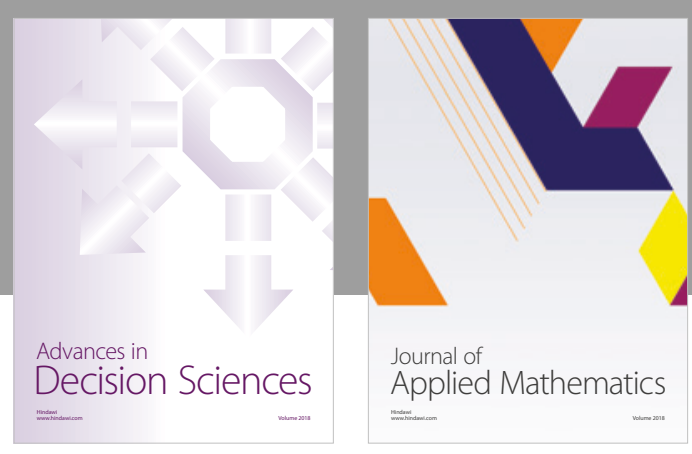

Journal of

Applied Mathematics
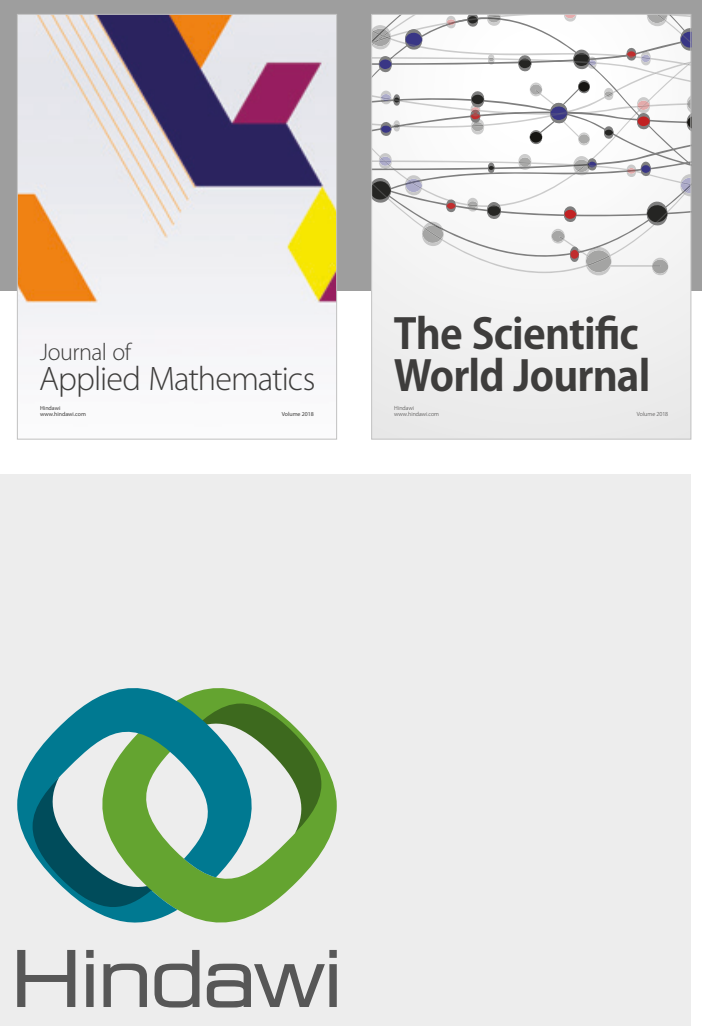

Submit your manuscripts at

www.hindawi.com

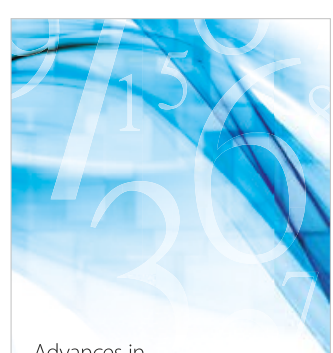

Advances in
Numerical Analysis
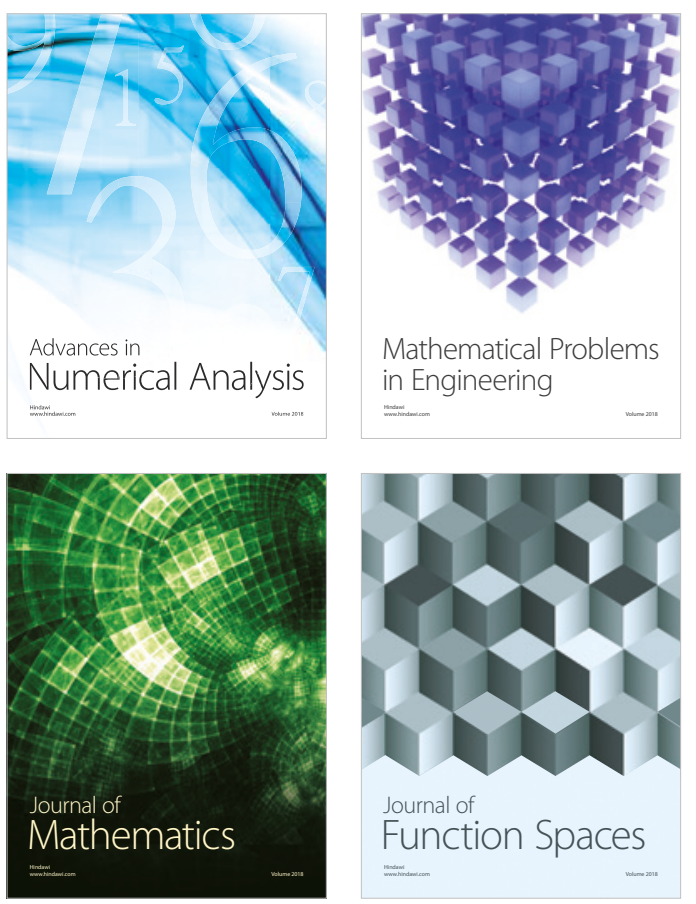

Mathematical Problems in Engineering

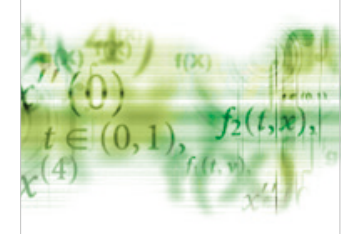

International Journal of

Differential Equations

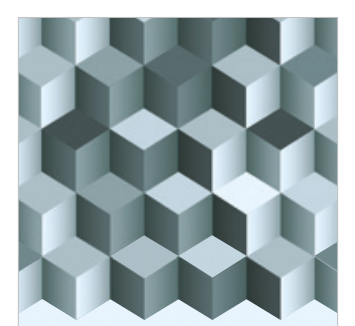

Journal of

Function Spaces

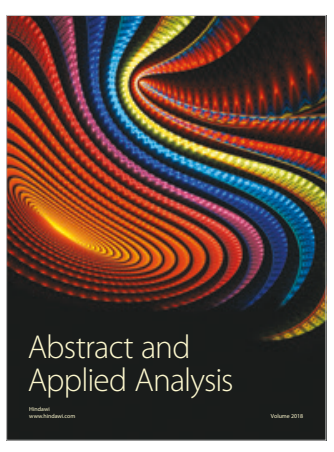

The Scientific

World Journal

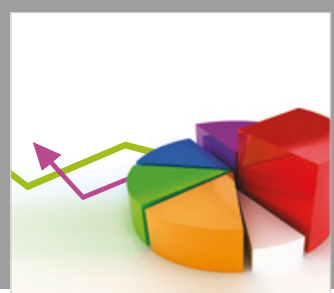

Journal of

Probability and Statistics
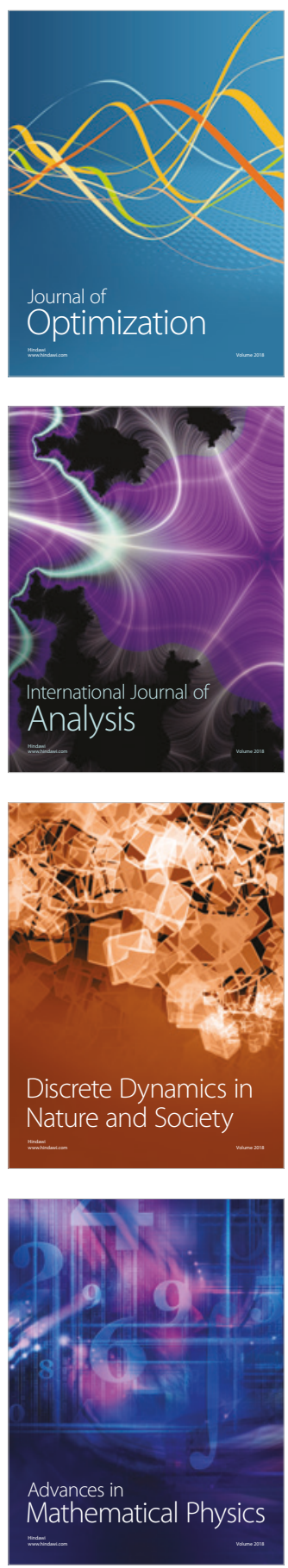